\title{
Arthropathies et côlite ulcéreuse
}

Les douleurs articulaires font partie du tableau classique de la recto-côlite ulcéro-hémorragique (R.-C. U.-H.); cependant, leur frequence est appréciée de façon très variée selon les pays: en France, Racket et Busson (collect. Flammarion à revision annuelle, p. 849) Testiment rare (2,5\% des cas), alors qu'aux U.S.A., Bockus (Gastroenterology, $2^{\circ}$ éd. [1964], vol. II. p. 848) en trouve 10 fois plus (22\%). Dans leur série de 269 R.-C. U.-H., V. Wright, rhumatologue, et G.

Watkínson, gastro-entérologue (Brit. med. J., pp. 670-675, 18 sept. 1965) relèvent des plaintes «rhumatoïdes» chez 45\% d'entre eux; mais chez les deux tiers, il s'agissait d'une douleur de la region sacrée (qui sera discutée plus bas) et les A. retiennent «seulement» 31 malades (ce qui fait encore $11,5 \%$ du total des côlitiques) atteints d'une arthropa-thie qu ${ }^{1 / 8} 1 \mathrm{~s}$ estíment spécífique et appellent «colitic arthritis».

10 Cette complication apparaît surtout entre 25 et 44 ans (exacte-ment comme Гâge de predilection de la R.-C. U.-H.) avec une frequence identique chez la femme et chez l'homme. Deux fois sur trois, cette arthrite commence de façon aiguë, simulant le rhumatisme arti-culaire aigu, et répondant d'ailleurs bien au salicylate. L'arthrite «cô-litique» se différencie de l'arthrite «rhumatoïde» par l'âge d'appari-tion (35 à 64 ans pour cette dernière), par l'atteinte monoarticulaire, surtout du membre inférieur (genou puis cheville), par Гabsence de nodules souscutanés ou d'inflammation péri-tendineuse. Enfin, la premiere poussée s'efface en general en moins de 6 semaines; mais elle récidive, d'habitude sur une articulation d'un autre membre, de sorte que les auteurs ont observe 71 episodes chez leurs 31 malades. Mais cette complication guérit avec restauration fonctionnelle complete presque constante ( 3 résidus objectivables sur 31 malades).

L'«arthrite côlitique» ne debute jamais avant l'apparition de phé-nomènes côlitiques, et les rechutes se produisent surtout dans les formes à evolution prolongée, soit périodique, soit d'une seule tenue; l'arthropathie est déclenchée souvent à la suite d'une exacerbation de la côlite, et s'efface en même temps que les symptômes intestinaux. II en est de même avec le traitement chirurgical applique à 15 malades : 5 d'entre eux se plaignirent encore de douleurs rhumatismales, mais il ne s'agissait jamais d'arthr_íe. Enfin (mais chez la femme 126

\section{Editorial}

seulement), les phénomènes articulaires sont plus frequents quand la R.-C. U.-H. s'étend à tout le colon; et dans les deux sexes elle correspond souvent avec d'autres complications: apbtes buccaux, uvéite et surtout dermatoses (erythème noueux, pyodermite, ulcères dits variqueux, etc.).

$2^{\circ} \mathrm{A}$ côté de cette complication articulaire, les mêmes médecins britanniques ont été frappés de la frequence de Tarthrite sacro-iliaque (A. S.-I.) chez leurs malades. Chez 234 R.-C. U.-H., ils ont compare radiologiquement la region sacro-iliaque (cliche de face) et la colonne lombaire (face et profil) avec les mêmes segments vertébraux de 234 témoins (Brit. med. J., pp. 675-680, 18 sept. 1965), et ils ont trouvé chez les premiers 84 cas de A. S.-I. contre 46 chez les témoins. Cette difference devient hautement significative quand on laisse de côté les formes bénignes et 
compare seulement les formes moyennes et sévères: on obtient alors $18 \%$ d'A. S.-I. dans la R.-C. U.-H., contre 4,7\% parmi les témoins. Heureusement, cette haute frequence radiologique ne se traduit pas toujours en clinique: il y avait seulement 9 spondy-lites ankylosantes (sur 85), 4 décalcifications ligamentaires; en revanche, on observa 18 amincissements des disques intervertébraux et 59 ostéophytoses (surtout chez les personnes âgées).

Les relations avec la côlite ne permettent pas d'incriminer un role preponderant des formes aiguës ou non, de Гextension des lesions ou de leur ancienneté; seule la rectite hémorragique localisée semble jouir d'une certaine immunité. Parmi 151 opérés, on ne retrouve plus qu'une seule A. S.-I. active (chez une femme de 21 ans). Enfin, s'il n'existe pas de relation entre l'A. S.I. et certaines complications de la R.-C. U.-H., on note cependant une relation très nette, mais chez la femme seulement, de l'A. S.-I. avec $\Gamma$ «arthrite côlitique» décrite ci-dessus.

L'A. S.-I. est en relation d'ailleurs avec bien d'autres affections intestinales que la R.-C. U.-H.: mentionnons l'iléite régionale, la dy-senterie bacillaire, la brucellose, la lipodystrophie de Whípple, etc.. . . Le fait que la spondylite peut précéder la R.-C. U.-H. exclut Thypo-thèse pathogénique d'une infection à point de depart côlique; on in-voque alors un facteur d'autoimmunité. .

On regrette, dans ces intéressants articles si bien documentés numériquement, Гabsence de precision sur la biologie de Гarthrite côlitique, et sur le traitement de ces deux complications fréquentes de la R.-C. U.-H. M. D. 